\title{
Acknowledgement to Reviewers of Viruses in 2015
}

\author{
Viruses Editorial Office \\ Published: 22 January 2016 \\ MDPI AG, Klybeckstrasse 64, CH-4057 Basel, Switzerland; viruses@mdpi.com
}

The editors of Viruses would like to express their sincere gratitude to the following reviewers for assessing manuscripts in 2015.

We greatly appreciate the contribution of expert reviewers, which is crucial to the journal's editorial decision-making process. Several steps have been taken in 2015 to thank and acknowledge reviewers. Good, timely reviews are rewarded with a discount off their next MDPI publication. By creating an account on the submission system, reviewers can access details of their past reviews, see the comments of other reviewers, and download a letter of acknowledgement for their records. In addition, MDPI has launched a collaboration with Publons, a website that seeks to publicly acknowledge reviewers on a per journal basis. This is all done, of course, within the constraints of reviewer confidentiality. Feedback from reviewers shows that most see their task as a voluntary and mostly unseen work in service to the scientific community. We are grateful to our reviewers for the contribution they make.

\begin{tabular}{lll} 
Abd-Alla, Adly & Hearing, Patrick & Peersen, Olve \\
Abdelwhab, E. M. & Heckmann, Josef G. & Peikert, Tobias \\
Abdul-Careem, M. F. & Hefferon, Kathleen Laura & Peloponese Jr., Jean-Marie \\
Abedon, Stephen & Heldwein, Ekaterina & Peng, Chien-fang \\
Abergel, Chantal & Helland, Today Emil & Pérez Ruiz, Mercedes \\
Aboulata, A. E. & Helle, Francois & Perez-Romero, Pilar \\
Abram, Michael & Henderson, Andrew & Peterschmitt, Michel \\
Accotto, Gian Paolo & Henke, Andreas & Petravic, Janka \\
Achazi, Katharina & Henzy, Jamie & Peyambari, Mahtab \\
Adelman, Zachary N & Herath, Tharangani & Pfeffer, Sébastien \\
Adler, Barbara & Hernalsteens, Jean-Pierre & Piantino Ferreira, AJ \\
Adlhoch, Cornelia & Hernandez-Alcoceba, Ruben & Pijlman, Groben \\
Adriaenssens, Evelien & Herrero, Salvador & Pique, Claudine \\
Adriana E., Kajon & Hewson, K. A. & Pise-Masison, Cynthia A. \\
Aebischer, Andrea & Hilgenfeld, Rolf & Plażuk, Damian \\
Aghemo, Alessio Michele & Hill, Andy & Pleschka, Stephan \\
Aguilar-Lemarroy, Adriana & Hirsch, Alec J. & Plevka, Pavel \\
Airenne, Kari & Hirsch, Ivan & Podhajcer, Osvaldo L. \\
Aires, Alfredo & Hishiki, Takayuki & Poehlmann, Stefan \\
Ait-Ali, Tahar & Hobman, Tom C. & Poelman, Randy \\
Alejo, Alí & Hobson-Peters, Jody & Polacek, Charlotta \\
Alemany, Ramon & Hoeben, Rob & Poli, Guido \\
\hline
\end{tabular}


Allander, Tobias

Almazan, Fernando

Alonso, Marta M.

Altan-Bonnet, Nihal

Alvarado, Julia Béjar

Alvarez, Ronald D.

Álvarez Sánchez, Julio

Alvisi, Gualtiero

Amadori, M.

Ananworanich, Jintanat

Andersson, Bjorn

Andrei, Graciela

Andreoletti, Laurent

Angelova, Assia

Ansaldi, Mireille

Anton, Josefa

Arif, Basil

Ariza, Maria Eugenia

Arnaud, Frederick

Arnold, Michelle

Ascencio-lbanez, Jose

Asfor, Amin S.

Azab, Walid

Azzouz, Mimoun

Bagamian, Karoun

Baird, Nicholas

Bajorek, Monika

Bakonyi, Tamas

Balfour, Henry

Bálint, Ádám

Balkema-Buschmann, Anne

Balvers, Rutger K.

Banfield, Bruce

Bangham, Charles R.M.

Bannert, Norbert

Bar-Joseph, Moshe

Baraka, Khaled

Barbu, Magda

Barik, Sailen

Barklis, Eric

Barrett, John

Barrett, Alan D. T.
Hoenen, Thomas

Holbrook, Michael R.

Hondan, Tomoyuki

Hong, Jiann-ruey

Honko, Anna

Horie, Masayuki

Horii, Takuro

Horimoto, Taisuke

Hrabal, Richard

$\mathrm{Hu}$, John

Huang, Jinghe

Huang, Chienjin

Huemer, Hartwig P.

Hufton, Simon E.

Hughes, Austin

Hughes, Grant

Humbel, Bruno

Hummel, Mary

Hunsperger, Elizabeth

Hust, Michael

Hutchinson, Edward C.

Huthoff, Hendrik

Ibanez, Trino Ascencia

Icenogle, Joseph

Ishikawa, Masayuki

Ishima, Rieko

Ito, Naoto

Iwanaga, Masashi

Izquierdo-Useros, Nuria

Jackson, Terry

Jackson, William

Jacobs, N.

Jacobson, Steven

Jahrling, Peter

Jain, Pooja

Jang, Ho Bin

Jehle, Johannes

Jeske, Holger

Johnson, Reed F.

Johnson, Nick

Jolly, Clare

Jones, Ian
Pompei, Raffaello

Pope, Welkin

Popham, Holly J.R.

Popham, Holly J.

Possee, Robert

Poveda, Eva

Powis, S. J.

Prangishvili, David

Prescott, Joseph

Préville, Xavier

Pyeon, Dohun

Qiao, Wentao

Quackenbush, Sandra

Quax, Tessa

Quer, Josep

Quinn, Thomas C.

Rabkin, Samuel D.

Racaniello, Vincent

Ragonnet-Cronin, Manon

Rajawat, Yogendra

Rakotondrafara, Aurelie

Randall, Troy D.

Rao, Venigalla B.

Ratner, Lee

Rauf, Abdul

Rautenschlein, Silke

Ravoet, Jorgen

Ray, Felix

Rees, Catherine

Reid, Steven

Reid, St. Patrick

Reid, Tony

Rein, Alan

Reperant, Leslie

Ressing, Maaike

Revilla, Yolanda

Rey, Chrissie

Ricciardi, Robert

Rice, Andrew

Richardson, Charles C.

Richert-Pöggeler, Katja

Rihn, Suzannah 


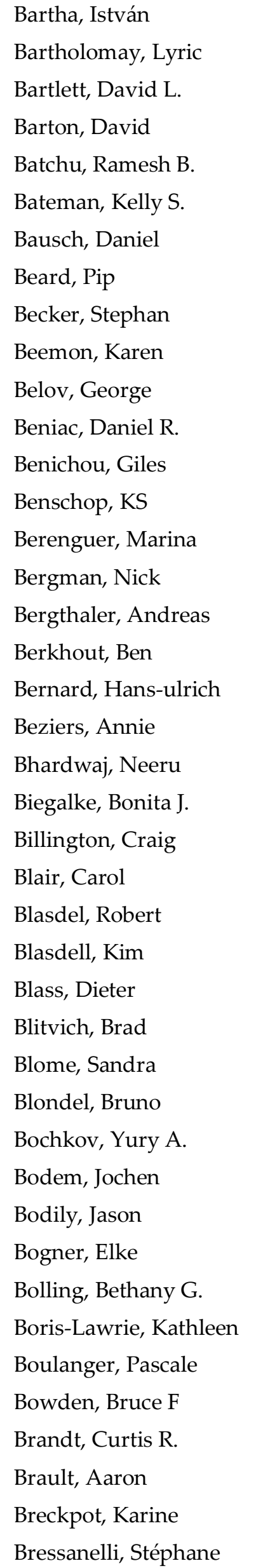

\begin{tabular}{|c|c|}
\hline Jones, Clinton & Rimstad, Espen \\
\hline Jones, Jeremy & Rinderer, Thomas E. \\
\hline Jones, Kathryn & Risatti, Guillermo R. \\
\hline Journo, Chloé & Risco, Cristina \\
\hline Junglen, Sandra & Rivas, C. \\
\hline Junichiro, Yasunaga & Robert-Guroff, Marjorie \\
\hline Kadowaki, Tatsuhiko & Roberts, Sally \\
\hline Kaguni, Jon M. & Robertson, Erle S. \\
\hline Kaida, Atsushi & Robinson, Gene \\
\hline Kajaste-Rudnitski, Anna & Roden, Richard \\
\hline Kalejta, Robert & Rodriguez, Jose F. \\
\hline Kalergis, Alexis M. & Rodriguez, Lorena \\
\hline Kalinke, Ulrich & Roh, Changhyun \\
\hline Kamen, Amine A. & Roine, Elina \\
\hline Kamil, Jeremy & Romalde, Jesús López \\
\hline Kanda, Tatsuo & Romanelli, Maria Grazia \\
\hline Kandathil, Abraham & Romanowski, Victor \\
\hline Kanduc, Darja & Romero-Brey, Inés \\
\hline Kang, Hyojeung & Roques, Pierre \\
\hline Kang, Congbao & Rosenberg, Helene \\
\hline Kariwa, Hiroaki & Rossetto, Cyprian C. \\
\hline Karlin, David G. & Rossi, John J. \\
\hline Karlsson, Erik & Rossman, Jeremy \\
\hline Kashanchi, Fatah & Rossmann, Michael G. \\
\hline Keil, Günther M. & Rota, Paul A \\
\hline Kennedy, Richard G. & Rothenburg, Stefan \\
\hline Kesmir, Can & Rowan, Aileen \\
\hline Khalifa, Mahmoud E & Rowlands, David J. \\
\hline Khan, Arifa & Roy, Polly \\
\hline Khan, Mazhar & Rubio, Luis \\
\hline Khan, Zafar K. & Ruggli, Nicolas \\
\hline Khatri, Mahesh & Russell, Rodney \\
\hline Khromykh, Alexander A. & Ryabov, Eugene V. \\
\hline Khromykh, Alexander & Ryabova, Lyuba \\
\hline Kielan, Margaret & Ryckman, Brent \\
\hline Kikuchi, Yutaka & Ryu, Sangryeol \\
\hline Kim, Kisoon & Saayman, Sheena \\
\hline Kim, Soohyun & Sadeuh-Mba, Serge \\
\hline Kimura, Takashi & Sadiq, S. Kashif \\
\hline Kindrachuk, Jason & Saelens, Xavier \\
\hline Kirchhoff, Frank & Sáiz, Margarita \\
\hline Kirkegaard, Karla & Saleh, Maria-Carla \\
\hline
\end{tabular}


Breyton, Cécile

Briant, Laurence

Bridle, Byram W.

Briers, Yves

Brighty, David

Brinkmann, Melanie

Brockmeier, Susan L.

Brooke, Christopher B

Brown, Jay

Bruessow, Harald

Buchholz, Christian J.

Buck, Christopher

Bugert, Joachim

Bull, Rowena

Bullitt, Esther

Büning, Hildegard

Burk, Robert D.

Burke, Don

Büttner, Mathias

Caballero, Primitivo

Cady, Kyle

Calisher, Charles

Cambillau, Christian

Campadelli-Fiume, Gabriela

Campbell, Edward

Camus-Bouclainville, Christelle

Candolfi, Marianela

Cañizares, Carmen

Cao, Yong-chang

$\mathrm{CAO}$, Pengxing

Caplen, Natasha J.

Carbonell, Alberto

Cardoso, Tereza C

Carfi, Andrea

Carpenter, Susan

Casasnovas, José M.

Casseb, Jorge

Cen, Osman

Cenens, William

Ceyssens, Pieter Jan

Chandran, Bala

Chang, Jinhong
Kiron, Viswanath

Kirsebom, Leif

Kitchen, Scott G.

Kitchen, Andrew

Klasse, Per Johan

Klein, Christian

Klose, Thomas

Klumpp, Jochen

Klupp, Barbara

Kobayashi, Kappei

Kok, Chee Choy

Kootstra, Neeltje

Korukluoglu, Gulay

Kousoulas, Konstantin G.

Kozak, Christine

Kranzusch, Philip

Krogstad, Paul

Kryger, Per

Kuhn, Richard

Kuhn, Jens

Kushibiki, Toshihiro

Kutter, Elizabeth

Kvarnheden, Anders

Lacomme, Dr. Christophe

Lacroix, Jeannine

Laethem, Kristel Van

Lafon, Monique

Lai, Michael M. C.

Laimins, Lou

Langereis, Martijn A.

Langlois, Ryan

Launes, Cristian

Lavazza, Antonio

Lavergne, Anne

Lavigne, Rob

Law, Manson

Lee, Sang-Won

Lee, Long Huw

LeGrice, Stuart

Lelli, Davide

Lemasson, Isabelle

Lemay, Guy
Sam Zhou, Heshan

Sambrie, Vittorio

Sandstrom, Paul

Sang, Yongming

Saphire, Erica

Sapp, Martin

Sargueil, Bruno

Sattar, Sampurna

Savenkov, Eugene

Sawa, Hirofumi

Scagnolari, Carolina

Schaap, Ivan

Schaar, Hilde M. Van Der

Schang, Luis

Scheibenbogen, Carmen

Schildgen, Oliver

Schleiss, Mark

Schmaljohn, Alan

Schmelcher, Mathias

Schmidt, Manfred

Schmidtke, Michaela

Schneider, Dirk

Schnell, Matthias J.

Schoborg, Robert V.

Schöning, Caspar

Schountz, Tony

Schröder, Martina

Schroeder, Declan C.

Schubert, Jörg

Schüpbach, Jörg

Scotch, Matthew

Seeger, Christoph

Sekine, $\mathrm{K}$

Sen, Adrish

Seong, Baik Lin

Serwer, Philip

Seto, Donald

Sevilla, Noemí

Shai, Yechiel

Shechter, David

Shelby, Kent S.

Sherer, Nathan M. 
Chang, Kuan Y.

Charpentier, Charlotte

Charrel, Rémi

Chejanovsky, Nor

Chen, Benjamin

Chen, Jason

Chen, Qi

Chen, Rong

Chen, $\mathrm{Li} \mathrm{Li}$

Chiu, Wah

Cho, Namhoon

Choi, Kyung H

Chong, Pele

Chow, Yen-Hung

$\mathrm{Chu}$, Dong

Chu, Justin

Chung, Amy

Ciccone, Nick A.

Cichero, Elena

Cicin-Sain, Luka

Ciotti, Marco

Clem, Rollie

Clement, Jan

Clementi, Nicola

Coen, Donald M.

Coffey, Lark L.

COHRS, RANDALL

Coiras, Mayte

Colberg-Poley, Anamaris

Connolly-Andersen, A. M.

Conrad, Nicholas

Conzelmann, Klaus

Conzelmann, Karl-Klaus

Cook, Atlanta G.

Cory, Jeny

Cosset, François-Loïc

Coutts, Robert H. A.

Coyne, Carolyn

Craigie, Robert

Croker, Ben

Crump, Colin

Csorba, Tibor
Leng, Qibin

Lever, Andrew

Levin, Henry L.

Li, Feng

Li, Xuguang

Liang, Chen

Liang, Zhenglun

Lichty, Brian D.

Liikanen, Llkka

Lin, Cheng-Wen

Lin, Rongtuan

Lin, Na-Sheng

Lindberg, A. Michael

Lindemann, Dirk

Lindh, Magnus

Ling, Kai-Shu

Linial, Maxine L.

Lippe, Roger

Liu, Wen

Liu, Shan-Lu

Liu, Dingxiang

Liu, Shuwen

Liu, Chia-Chyi

LiWang, Patricia

Loessner, Martin

Lohmann, Volker

Lokensgard, James $\mathrm{R}$

López, Daniel

López-Ferber, Miguel

Lossius, Andreas

Luciani, Fabio

Luftig, Micah

Lukac, David

Lukashevich, Igor

Lundstrom, Kenneth

Lünemann, Jan D.

Luo, Shuhong

Luo, Dahai

$\mathrm{Ma}$, Wenjun

Macchi, Beatrice

Macdonald, Andrew

Machamer, Carolyn
Shi, ZhengLi

Shisler, Joanna

Silbart, Lawrence K.

Siliciano, Robert F.

Simón, Oihane

Sinclair, John

Sinkins, Steven

Skalka, Anna Marie

Skalsky, Rebecca

Skinner, Mike

Slezak, Tom

Smerdou, Christian

Smit, Sandra

Smith, Kellie N

Smith, Donald

Smith, David W.

Smith, Darren

Smith, Darci

Smith, Jason G.

Smith, Robert A.

Smithgall, Thomas E.

Snyder, Christopher M.

Sobrino, Francisco

Sodeik, Beate

Song, Dae-Sub

Song, Jiuzhou

Sönnerborg, Anders

Sorokulova, Iryna

Sozhamannan, Shanmuga

Spencer, Juliet V.

Spilki, Fernando

Stamminger, Thomas

Stanton, Richard

Stanton, Robert C.

Stanway, Glyn

Steinmann, Eike

Stenglein, Mark

Stephens, Edward

Stern, Peter

Steven, Alasdair C.

Stewart, Meredith

Stice, Steve L. 
Dąbrowska, Krystyna

Dainat, Benjamin

Dalianis, Tina

Dalmo, Roy A

Davenport, Miles

Dawson, Christopher W.

Day, Patricia M.

Day, James Michael

De Francesco, Raffaele

de Graaf, Daniel

De La Torre, Juan Carlos

De Los Santos, Teresa

de Miranda, Joachim

de Motes, Carlos Maluquer

de Pablo, Pedro

De Silva, Aravinda M.

Debarbieux, Laurent

Debyser, Zeger

DeFilippis, Victor R.

Delang, Leen

Delmas, Bernard

Denner, Joachim

Deom, Carl

Desbiez, Cécile

Dhurandhar, Nikhil

Diallo, Jean-Simon

Diamond, Don J.

Díaz Pendón, Juan Antonio

Diedrich, Sabine

Diefenbach, Russell J.

Dietzgen, Ralf G.

Diez, Juana

Dimaio, Daniel

Dimitrov, Dimiter

Dobner, Thomas

Dobrovolny, Hana M.

Dolei, Antonina

Dolnik, Olga

Dominguez, Samuel

Donato, Celeste

Doorbar, J

Doublet, Vincent
MacLachlan, James

Madan, Vanesa

Maecker, Holden

Maekawa, Shinya

Magae, Junji

Mager, Dixie

Mahony, Timothy John

Makinen, Kristiina

Maldarelli, Frank

Mameli, Giuseppe

Mancini, Nicasio

Marc, Daniel

Marcello, Alessandro

Marchini, Antonio

Marcotrigiano, Joseph

Maria Isabel, Sola Gurpegui

Maria Vaira, Anna

Marjomäki, Varpu

Marjuki, Henju

Markov, Peter V.

Markovitz, David

Marques, Joao

Marsh, Mark

Marshall, John A.

Martinez, Osvaldo

Martínez, Isidoro

Martínez-Salas, Encarnación

Martinez-Sobrio, Luis

Marzano, Shinyi

Masson, Jean

Matranga, Chris

Matsukura, Keiichiro

Matthews, Stephen

Maury, Wendy

Mayer, Jens

Mazurov, Dmitriy V

McBride, Alison

McCance, Dennis

McCauley, John W.

Mcclure, Myra

McCormick, Craig

McElroy, Anita
Stojdl, David

Stoltzfus, C. Martin

Stonehouse, Nicola

Stoye, Jonathan

Strick, Reiner

Strong, Jim

Strong, James

Sui, Guangchao

Sullivan, Deborah E.

Sullivan, Chris

Sullivan, Matthew B.

Sun, Bing

Sun, Ren

Susi, Petri

Suzuki, Jon

Suzuki, Tohru

Svitkin, Yuri

Swamy, Manjunath N.

Swanson, Chad

Swigon, David

Switzer, William

Tagaya, Yutaka

Takeda, Shigeki

Takegami, Tsutomu

Takeuchi, Yasuhiro

Takimoto, Toru

Tamori, Akihiro

Tanaka, Yuetsu

Tang, Hengli

Tedbury, Philip

Tee, Kok-Keng

Tempesta, Maria

Terajima, Masanori

Tesh, Robert B.

Theilmann, David

Théry, Clotilde

Thézé, Julien

Thorne, Stephen H.

Tietjen, Ian

Tikoo, Suresh K.

Tommasino, Massimo

Tommassino, Massimo 


\begin{tabular}{|c|c|c|}
\hline Dräger, Carolin & McFadden, Grant & Torres, Jaume \\
\hline Dreux, Marlene & McInerney, Gerald & Travé, Gilles \\
\hline Drexler, Jan Felix & Mcinnes, Colin & Trobridge, Grant D. \\
\hline Dropulić, Boro & McLaughlin-Drubin, Margaret & Tsai, Billy \\
\hline Drosten, Christian & Mcminn, Peter C. & Tsai, C.H. \\
\hline Drulis-Kawa, Zuzanna & Mcnamara, James & Tsukiyama-Kohara, Kyoko \\
\hline Dubuisson, Jean & McTaggart, Seanna J & Turnbull, Matthew W. \\
\hline Duerksen-Hughes, Penelope J. & Medina, Rafael A. & Ueda, Koji \\
\hline Dvorak, Cheryl & Meeus, Ivan & Ueda, Keiji \\
\hline Edgar, James & Meijer, Adam & Uprichard, Susan L \\
\hline Eichwald, Catherine & Meins, Frederick Jr. & Urcelay, Elena \\
\hline El Zowalaty, Mohamed & Melero, José A. & Vähä-Koskela, Markus \\
\hline Elbeaino, Toufic & Mellors, John W. & Vahidnia, Farnaz \\
\hline Elder, John H. & Mendoza-Cano, Fernando & Valles, Steven M. \\
\hline Elshabrawy, Hatem & Menendez-Arias, Luis & Vallinoto, Antonio \\
\hline Emerson, Ginny & Meneses, Patricio I. & van den Ham, Henk-Jan \\
\hline Enjuanes, Luis & Mercer, Andrew & Van Den Hoogen, B. G. \\
\hline Enquist, Lynn W. & Mercer, Jason & Van Den Hurk, Andrew \\
\hline Ensser, Armin & Mergia, Ayalew & Van der Kuyl, Antoinette \\
\hline Eriksson, Kristina & Merits, Andres & van der Poel, Wim \\
\hline Erlandson, Martin & Meseda, Clement & Van Duyne, Rachel \\
\hline Errington-Mais, Fiona & Metcalf, Jordan P. & Van Etten, James \\
\hline Essani, Karim & Metzner, Karin & Van Grevenynghe, Julien \\
\hline Etebari, Kayvan & Meyers, Gregor & van Hoek, B. \\
\hline Evans, Matthew & Michiels, Thomas & van Lier, René \\
\hline Everett, Roger D. & Millard, Andrew & Van Raaij, Mark \\
\hline Favier, Anne-Laure & Miller, Sara E & Van Sinderen, Douwe \\
\hline Federico, Maurizio & Miller, Stefan & Vander Hoek, Lia \\
\hline Feng, Pinghui & Miller, George & Vaneechoutte, Mario \\
\hline Ferrante, Pasquale & Miller, E. Kathrin & Vanham, Guido \\
\hline Ferrari, Guido & Minsky, Abraham & Varzakas, Theodoros \\
\hline Fielding, Burtram C. & Mintz, Paul & Vassilopoulos, George \\
\hline Filippov, Valery & Mlotshwa, Sizolwenkosi & Veluthambi, Karuppanan \\
\hline Fillat, Cristina & Mochizuki, Tomofumi & Venuti, Aldo \\
\hline Finke, Stefan & Monier, Adam & Verbeek, Martin \\
\hline Fischer, Wolfgang B. & Moody, Cary & Verdaguer, Nuria \\
\hline Fisher, Chris & Moore, Sean D. & Verheije, Hélène \\
\hline Fleming, Steve & Moreau, Hervé & Verheyen, Jens \\
\hline Flenniken, Michelle & Morgan, Iain & Verma, Subhash C \\
\hline Flint, Jane & Mori, Seiichiro & Vidal, Silvia \\
\hline Flodström-Tullberg, Malin & Morrical, Scott W. & Vieillard, Vincent \\
\hline Flores, Ricardo & Morrison, Richard & Vilches, Carlos \\
\hline
\end{tabular}


Flores, Eduardo F

Florin, Luise

Foley, Brian T.

Forlenza, Maria

Fouts, Derrick E

Freed, Eric $\mathrm{O}$.

Frensing, Timo

Friedland, Robert

Friedman, Gregory K.

Frieman, MB

Frisk, Gun

$\mathrm{Fu}$, Zhen

Fuentes, Alejandro

Fukushi, Hideto

Gallagher, Tom

Gallay, Phillip

Ganges, Llilianne

Gao, Feng

Gara, Naveen

García, Maricarmen

Garcia, Pilar

Garcia-Estañ, Luis Perez

Garozzo, Adriana

Garrote, José Ignacio Núñez

Gatignol, Anne

Geisbert, thomas w

Genersch, Elke

Georgel, Philippe

Gessain, Antoine

Ghany, Marc

Ghose, Ranajeet

Giam, Joe

Gifford, Robert J.

Gilbert, Caroline

Gilbert, Clément

Gillet, Nicolas

Gillet, Laurent

Gisder, Sebastian

Goffard, Anne

Goldmana, Ellen R.

Goldsmith, Cynthia

Golovanov, Alexander P.
Morrison, Trudy G

Mortreux, Franck

Morya, Vivek

Moseley, Gregory

Moss, Bernard

Mothes, Walther H.

Mourier, Tobias

Mueller, Thomas

Mukhopadhyay, Suchetana

Mulder, Lubbertus

Mulkidjanian, Armen

Muller, Claude P.

Münch, Jan

Munster, Vincent

Munster, Vincent J.

Murata, Takayuki

Murphy, Lita

Mussolino, Claudio

Muylkens, Benoît

Mymryk, Joseph

N. Frick, David

Naesens, Lieve

Nagata, Noriyo

Nagy, Peter D.

Nájera, Isabel

Nakahara, Kenji S.

Nekhai, Sergei

Nelson, Kenrad

Netherton, Christopher L.

Nettelbeck, Dirk M.

$\mathrm{Ng}$, Lisa F.P.

Nicoletti, Loredana

Nieto, Amelia

Nilsson, Anders

Nisole, Sebastien

Nitsche, Andreas

Noda, Hiroaki

Nolasco, Gustavo

Norder, Heléne

Novella, Isabel

Nowotny, Norbert

Nunes, Márcio R. T.
Villalain, Jose

Visseaux, Benoit

Vitour, Damien

Vlak, Just M.

Vogel, Franziska

Volchkova, Valentina

von Hahn, Thomas

Waheed, Abdul A.

Wainberg, Mark

Wakimoto, Hiroaki

Walker, Thomas

Wan, Yonghong

Wanasen, Nanchaya

Wang, Qian

Wang, Shu

Wang, Jieru

Wang, Robert Y.L.

Wang, Chi-Young

Wang, Jen-ren

Wang, Songjie

Wang, Ying

Wang, Ching-ho

Wang, Han-Ching

Wang, Pei Gang

Warfield, Kelly L.

Waris, Gulam

Watanabe, Toshiki

Weber, Irene

Węgrzyn, Grzegorz

Wei, Xierong

Wei, Yangdou

Weinman, Steven

Weissenhorn, Winfried

Weitzman, Matthew

Weller, Sandra

White, Kirsten

Whitehurst, Christopher

Wiebe, Matthew S.

Wileman, Tom

Wilen, Craig

Wilhelm, Barbara

Willems, Luc 
Gong, Peng

Goodrum, Felicia

Gorbalenya, Alexander E.

Gorini Da Veiga, Ana

Gossner, Celine

Götte, Matthias

Graham, Sheila

Gramberg, Thomas

Grandgenett, Duane P.

Grandvaux, Nathalie

Grattagliano, Ignazio

Grdzelishvili, Valery Z.

Greber, Urs F.

Green, Patrick L.

Gregoire, Marc

Griffiths, Paul

Griffiths, Anthony

Gronenborn, Bruno

Grzywacz, David

Guillon, Christophe

Gummuluru, Suryaram

Gundogdu, Ozan

Guo, Z. Sheng

Gupta, Rashmi

Gupte, Sachin

Gustin, Jean K.

Gustin, Kurt

Haas, Juergen G.

Hajimorad, Reza

Hakami, Ramin

Hall, Roy

Han, Guan-zhu

Harris, Katharine

Harrison, Robert

Harrod, Robert

Hartshorn, Kevan

Hayward, S. Diane

He, Mingliang
Nuttall, Patricia A

O'connor, David H.

Obar, Joshua J.

Oberste, Steven

Obrępalska-Stęplowska, A.

Ohlmann, Théophile

Ohshima, Takayuki

Okazaki, Katsunori

Okino, Cintia Hiromi

Olinger, Gene

Oliver, Stefan

Olson, Kenneth

Ono, Akira

Ornelles, David

Ortego, Javier

Oshitani, Hiroshi

Ow, Thomas

Ozbun, Michelle

Pagano, Joseph S.

Pager, Cara

Palamara, Anna Teresa

Palese, Peter

Palmarini, Massimo

Paludan, Søren R.

Pan, Qiuwei

Panchal, Rekha G.

Pandha, Hardev

Papic, Neven

Pappu, Hanu

Paradkar, Prasad

Parent, Leslie

Parent, Kristin N.

Parish, Joanna L.

Pathak, Vinay

Patil, Basavaprabhu

Pattnaik, Asit

Patton, John

Peden, Keith
Williams, Trevor

Wills, John

Wilson, Van

Wimley, William C.

Wimmer, Eckard

Witcher, Michael

Wittmann, Jürgen

Wong, Sek Man

Wood, Henry M.

Worgall, Stefan

Workenhe, Samuel

$\mathrm{Wu}$, Yuntao

$\mathrm{Xi}$, Zhiyong

Yaegashi, Hajime

Yamanaka, Atsushi

Yamauchi, Yohei

Yang, Chinglai

Yang, Chunfu

Yang, Decheng

Yang, Feng

Yedavalli, Venkat

Yeh, Shyi-Dong

Yonekura, Kentaro

Yu, Xiao-Fang

Yuan, Weiming

Zakhartchouk, Alexander

Zamarin, Dmitriy

Zell, Roland

Zeltins, Andris

Zerbini, Francisco Murilo

Zhang, Yanjin

Zhang, Lian-feng

Zhang, Jianqiang

Zhang, Qijing

Zheng, Yong-Hui

Zhou, Peng

Zur Hausen, $\mathrm{H}$. 